\title{
Estimativa da resistência e da rigidez à compressão paralela às fibras da madeira de Pinus sp. pela colorimetria
}

\author{
Estimation of strength and stiffness in compression \\ parallel to the grain of Pinus sp. by colorimetry
}

\section{Lucas José Marini \\ Tiago Hendrigo Almeida \\ Diego Henrique Almeida \\ André Luis Christoforo \\ Francisco Antonio Rocco Lahr}

\section{$\mathbf{P}$}

ropriedades de resistência e de rigidez da madeira, fundamentais em um projeto estrutural, são determinadas com base em ensaios mecânicos. Entre as propriedades mecânicas da madeira, destacamse as de resistência $\left(\mathrm{f}_{\mathrm{c} 0}\right)$ e de rigidez $\left(\mathrm{E}_{\mathrm{c} 0}\right)$ à compressão na direção paralela às fibras. A cor é uma característica própria de cada espécie. Se determinadas experimentalmente as propriedades $\mathrm{f}_{\mathrm{c} 0}$ e $\mathrm{E}_{\mathrm{c} 0}$ de uma espécie de madeira com base em um significativo número de amostras caracterizadas, a correlação dessas propriedades por meio de equações obtidas por regressão possibilita realizar estimativas de tais propriedades sem a necessidade de novos ensaios, procedimento esse com potencial de aplicação por empresas madeireiras. Com o auxílio do sistema CIE L*a*b* e de 403 peças de madeira de Pinus sp., equações obtidas por modelo de regressão foram utilizadas para a estimativa do $\mathrm{E}_{\mathrm{c} 0}$ e da $\mathrm{f}_{\mathrm{c} 0}$ em função dos parâmetros colorimétricos e do número de anéis de crescimento. Da estimativa do $\mathrm{E}_{\mathrm{c} 0}\left(\mathrm{R}^{2}=43,61 \%\right)$, pela ANOVA da equação de regressão foi constatada significância apenas do número de anéis de crescimento (Nle). Para a resistência à compressão paralela às fibras $\left(\mathrm{R}^{2}=48,53 \%\right)$, apenas os parâmetros colorimétricos e as interações desses parâmetros afetaram significativamente a $\mathrm{f}_{\mathrm{c} 0}$.

${ }^{1}$ Lucas José Marini Universidade Federal de São Carlos São Carlos - SP - Brasil

${ }^{2}$ Tiago Hendrigo Almeida ${ }^{2}$ Instituto SENAI de Inovação Biomassa Três Lagoas - MS - Brasil

${ }^{3}$ Diego Henrique Almeida ${ }^{3}$ Universidade Federal de Rondônia Porto Velho - RO - Brasil

${ }^{4}$ André Luis Christoforo ${ }^{4}$ Universidade Federal de São Carlos São Carlos - SP - Brasil

${ }^{5}$ Francisco Antonio Rocco Lahr ${ }^{5}$ Universidade de São Paulo São Carlos - SP - Brasil

Recebido em 23/10/19 Aceito em 14/04/20
Palavras-chave: Propriedades mecânicas. Anéis de crescimento. Regressão múltipla.

\section{Abstract}

Strength and stiffness properties of the wood, fundamental in a structural design, are determined based on mechanical tests. Among the mechanical properties of wood, the strength $\left(f_{c 0}\right)$ and stiffness $\left(E_{c 0}\right)$ to compression in the parallel direction to the grain. Color is a characteristic of each species. If the $f_{c 0}$ and $E_{c 0}$ properties of a wood species are determined experimentally, based on a significant number of characterized samples, the correlation of these properties through equations obtained by regression makes it possible to estimates of such properties without the need for new tests, a procedure with potential for application by companies of the wood industry. With the aid of the CIE $L^{*} a^{*} b^{*}$ system and 403 pieces of wood from Pinus sp. equations obtained by regression analysis were used to estimate the $E_{c 0}$ and $f_{c 0}$ as a function of colorimetric parameters and also the number of growth rings. From the estimate of $E_{c 0}\left(R^{2}=43.61 \%\right)$, by ANOVA of the regression equation, it was found significance only in the number of growth rings (Nle).

For the compressive strength parallel to the grain $\left(R^{2}=48.53 \%\right)$, only the colorimetric parameters and the interactions of these parameters significantly affected the $f_{c o}$.

Keywords: Mechanical properties. Growth rings. Multiple regression. 


\section{Introdução}

A madeira está presente no cotidiano das pessoas nas mais diversas formas de utilização, tais como fabricação de móveis, celulose e papel, geração de energia e, especificamente para o escopo deste trabalho, na construção civil (ANDRADE JR. et al., 2014; ALMEIDA et al., 2017).

Para suprir a demanda por madeira serrada foi introduzido no Brasil o gênero Pinus, que conta com mais de cem espécies e é nativo do hemisfério Norte (EMPRESA..., 2018). Atualmente, no Brasil, as plantações de Pinus ocupam 1,6 milhão de hectares (INSTITUTO..., 2018).

Uma das principais características do gênero Pinus sp. é a presença de anéis de crescimento, que se originam da atividade sazonal do câmbio vascular, que é a estrutura interna responsável pelo crescimento radial das árvores. Em países onde o clima tem uma clara distinção entre as estações do ano é mais fácil identificar o início e o fim de um período de crescimento da árvore (FERREIRA, 2009).

Em cada anel de crescimento é possível encontrar duas regiões distintas, uma apresentando coloração clara e outra escura. O lenho inicial corresponde ao crescimento da árvore no início do período vegetativo, normalmente primavera e verão, em que há células de paredes finas e lumes grandes, o que lhe confere coloração clara e densidade menor. O lenho tardio é formado durante o outono e o inverno, sendo constituído de células com paredes mais largas e menor lúmen, uma vez que as células vão diminuindo a atividade fisiológica. Em consequência desse fato, sua coloração é escura e sua densidade é maior do que a do lenho inicial (SANTINI; HASELEIN; GATTO, 2000; BURGER; RICHTER, 1991).

O comportamento do material quando submetido a esforços varia de acordo com as propriedades mecânicas da madeira. $\mathrm{O}$ ensaio de compressão paralela às fibras é um dos mais importantes, uma vez que o valor característico da resistência à compressão paralela às fibras $\left(\mathrm{f}_{\mathrm{c} 0, \mathrm{k}}\right)$ possibilita o enquadramento da madeira nas classes de resistência estabelecidas pela NBR 7190 (ABNT, 1997), além de possibilitar a estimação do módulo de elasticidade $\left(\mathrm{E}_{\mathrm{c} 0}\right)$, que determina a rigidez da madeira utilizada no projeto (BERTOLINI et al., 2012; FERRO et al., 2015; ALMEIDA, 2017).

A coloração é uma propriedade organoléptica da madeira, que em conjunto com as propriedades anatômicas permite a identificação de espécies, exercendo também extrema importância sobre o valor decorativo do material, por adicionar valor estético. Pode ser influenciada por diversos fatores, tais como espécie, estrutura anatômica, composição química, genética, densidade e tratamentos silviculturais (MONTES et al., 2008; GARCIA; MARINONIO, 2016; GIERLINGER et al., 2003; GARCIA et al., 2014).

É importante ressaltar a subjetividade da propriedade cor, pois existem diferenças de sensibilidade dos observadores (ZENID; CECCANTINI, 2007). O modo encontrado para padronizar essa propriedade foi transformá-la em números. A Comissão Internacional de Iluminantes (CIE) recomendou o sistema CIE L*a*b* para padronizar a sensação de cor baseada nos parâmetros colorimétricos luminosidade e tonalidade, um dos sistemas mais utilizados para quantificação da cor (CAMARGOS; GONÇALEZ, 2001; ALMEIDA, 2017).

A técnica de colorimetria, que consiste em determinar a cor de uma superfície por meio de espectrofotômetros e colorímetros, tem sido aplicada à madeira para caracterizar seus parâmetros colorimétricos, avaliar os efeitos de tratamentos termomecânicos na coloração da madeira e avaliar infestações patológicas (AMORIM; GONÇALEZ; CAMARGOS, 2013; ZANUNCIO et al., 2014; CADEMARTORI et al., 2014; BARCÍK; GASPARÍK; RAZUMOV, 2015; POUBEL et al., 2015; MEINTS et al., 2016; LAZAROTTO et al., 2016). É uma técnica de simples aplicação, podendo ser empregada na pré-classificação a partir do desdobro da tora, na qualificação das peças e na formação de banco de dados (BARROS; MUNIZ; MATOS, 2014). Entretanto, são poucas as pesquisas desenvolvidas em que as propriedades mecânicas da madeira foram correlacionadas com os parâmetros colorimétricos (ALMEIDA, 2017). A vantagem no uso dessa abordagem alternativa ante os métodos destrutivos de caracterização da madeira está na possibilidade de analisar um grande volume de madeira com maior rapidez.

Dessa forma, o objetivo desta pesquisa consistiu em empregar modelos para gerar equações por meio de análise de regressão múltipla a fim de investigar a precisão desses na estimativa de propriedades de resistência e de rigidez na compressão paralela às fibras da madeira de Pinus sp. em função dos parâmetros colorimétricos $\left(\mathrm{L}^{*} \mathrm{a} \mathrm{b}^{*}\right)$ e do número de lenhos (inicial e tardio), de forma a identificar os fatores mais significativos.

150 Marini, L. J.; Almeida, T. H.; Almeida, D. H.; Christoforo, A. L.; Lahr, F. A. R. 


\section{Material e métodos}

Um lote homogêneo de madeira (mesma origem) do gênero Pinus foi empregado nesta pesquisa. Por estar a madeira convenientemente estocada, o teor de umidade foi próximo a $12 \%$, porcentagem que é a umidade de equilíbrio higroscópico estabelecida pela norma brasileira NBR 7190 (ABNT, 1997). A partir do lote foram confeccionados 403 corpos de prova com dimensões nominais de $50 \mathrm{~mm}$ de espessura, $100 \mathrm{~mm}$ de largura e $150 \mathrm{~mm}$ de comprimento (Figura 1) para a realização dos ensaios de compressão na direção paralela às fibras. Decidiu-se aumentar significativamente a amostragem para dar maior representatividade aos resultados obtidos, pois é desconhecida a relação entre os parâmetros colorimétricos e as propriedades mecânicas aqui investigadas para a madeira de Pinus.

\section{Ensaio de compressão paralela às fibras}

Os ensaios de compressão paralela às fibras foram realizados na máquina universal de ensaios Amsler, que possui capacidade de carga de 25 t. Iniciou-se a aplicação do carregamento monotônico pela máquina de ensaio com taxa crescente de $10 \mathrm{MPa} / \mathrm{min}$, de acordo com a norma NBR 7190 (ABNT, 1997). O ensaio foi automatizado utilizando-se dois medidores de deslocamentos e uma célula de carga com capacidade de $25 \mathrm{t}$ ligada a um sistema de aquisição de dados (Figura 2). Esse sistema foi configurado para coletar as informações de força e deslocamento a cada $0,5 \mathrm{~s}$.

Figura 1 - Corpo de prova antes do ensaio de compressão

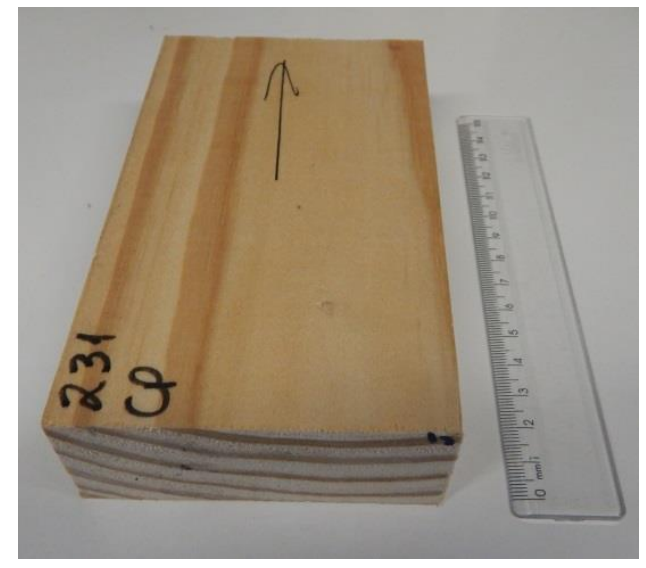

Figura 2 - Equipamento de ensaio de compressão paralela às fibras com corpo de prova, medidores de deslocamentos e célula de carga

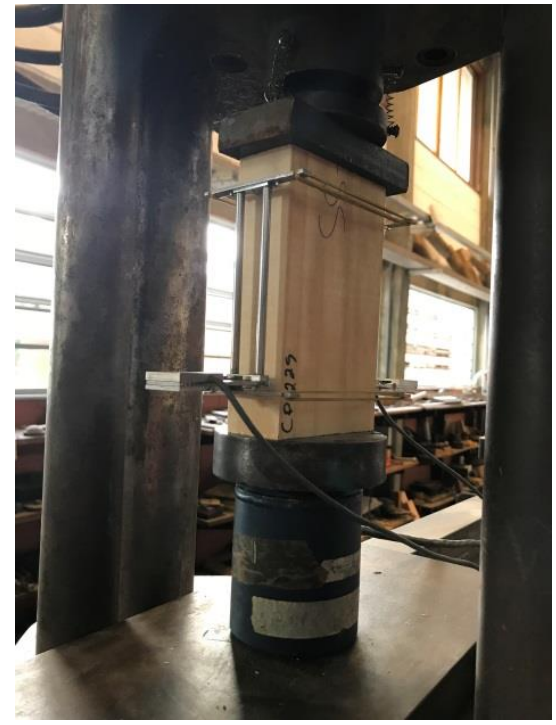


A resistência da madeira na compressão paralela às fibras $\left(\mathrm{f}_{\mathrm{c} 0}\right)$ é determinada pela relação entre a máxima força de compressão $\left(\mathrm{F}_{\mathrm{c} 0 \text {,máx }}\right)$ e a área da seção transversal $(\mathrm{A})$, como expressa a Equação 1.

$f_{c 0}=\frac{F_{c 0, m a x}}{A}$

Eq. 1

O módulo de elasticidade na compressão paralela $\left(\mathrm{E}_{\mathrm{c} 0}\right)$ foi obtido com o uso da Equação 2, em que $\sigma_{10 \%} \mathrm{e}$ $\sigma_{50 \%}$ são as tensões de compressão correspondentes a $10 \%$ e $50 \%$ da resistência $\mathrm{f}_{\mathrm{c} 0}$, e $\varepsilon_{10 \%}$ e $\varepsilon_{50}$ consistem nas deformações específicas correspondentes às tensões de $10 \%$ e $50 \%$.

$E_{c 0}=\frac{\sigma_{50 \%}-\sigma_{10 \%}}{\varepsilon_{50 \%}-\varepsilon_{10 \%}}$

Eq. 2

\section{Determinação dos parâmetros colorimétricos}

Os ensaios para determinação dos parâmetros colorimétricos foram realizados com um colorímetro da marca Konica Minolta, modelo CR-400, configurado com iluminante D65 e ângulo de observação de $10^{\circ}$, baseado no sistema CIE L*a*b* (Figura 3). Esse sistema consiste em três coordenadas: L*, que representa a luminosidade no eixo branco-preto, variando de 0 (preto) a 100 (branco); a* e b *, que representa as coordenadas cromáticas nos eixos verde-vermelho e amarelo-azul respectivamente. Ambos variam de -60 a +60 (Figura 4). O dispositivo de leitura posicionado sobre a superfície da madeira é circular, com $50 \mathrm{~mm}$ de diâmetro, e se ajustou perfeitamente às dimensões dos corpos de prova (Figura 3a). Nessas condições, os lenhos inicial e tardio são considerados em uma única leitura de imagem.

\section{Determinação do número anéis de crescimento (Nle)}

O anel de crescimento é composto de duas camadas, a primeira com tonalidade mais clara (lenho inicial), e a segunda de tonalidade mais escura (lenho tardio). Foram identificados visualmente os lenhos inicial e tardio nas duas seções transversais de cada corpo de prova (Figura 5).

\section{Análise estatística}

Modelo de regressão (Equação 3) avaliado pela análise de variância (ANOVA), ao nível de 5\% de significância, foi utilizado para investigar a influência dos parâmetros colorimétricos $\left(L^{*}, a^{*}, b^{*}\right)$ e do número de lenhos $(\mathrm{Nle})$ nos valores do módulo de elasticidade $\left(\mathrm{E}_{\mathrm{c} 0}\right)$ e na resistência $\left(\mathrm{f}_{\mathrm{c} 0}\right)$ à compressão na direção paralela às fibras da madeira de Pinus sp., utilizando-se 403 determinações para cada propriedade.

$\mathrm{Y}=\alpha_{0}+\alpha_{1} \cdot \mathrm{L}+\alpha_{2} \cdot \mathrm{a}+\alpha_{3} \cdot \mathrm{b}+\alpha_{4} \cdot \mathrm{Nle}+\alpha_{5} \cdot \mathrm{L} \cdot \mathrm{a}+\alpha_{6} \cdot \mathrm{L} \cdot \mathrm{b}+\alpha_{7} \cdot \mathrm{L} \cdot \mathrm{Nle}+\alpha_{8} \cdot \mathrm{a} \cdot \mathrm{b}+\alpha_{9} \cdot \mathrm{a} \cdot \mathrm{Nle}+\alpha_{10} \cdot \mathrm{b} \cdot \mathrm{Nle}+\varepsilon \quad$ Eq. 3

\section{Figura 3 - Colorímetro portátil Konica Minolta}

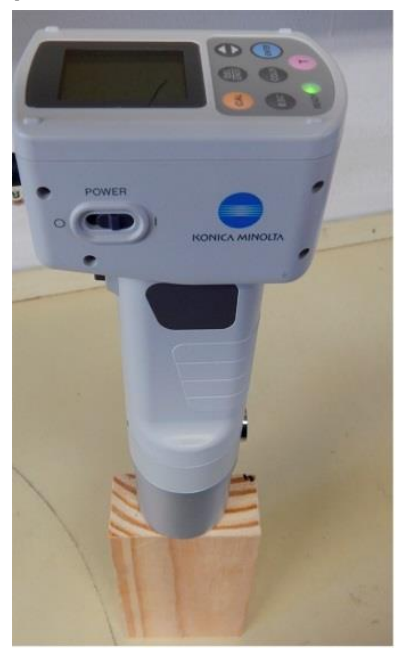

(a) Ensaio sendo realizado na amostra

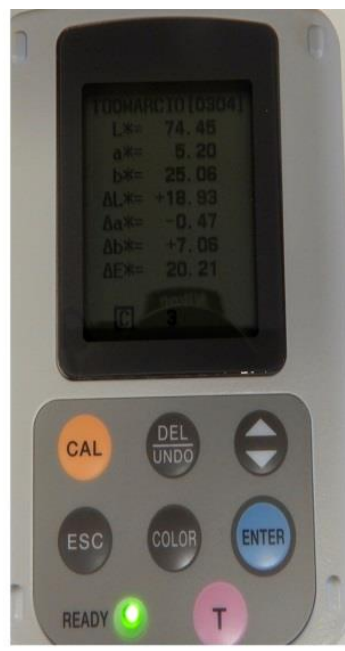

(b) Visor do aparelho com os valores dos parâmetros colorimétricos

152 Marini, L. J.; Almeida, T. H.; Almeida, D. H.; Christoforo, A. L.; Lahr, F. A. R. 
Figura 4 - Sistema CIE L*a*b*

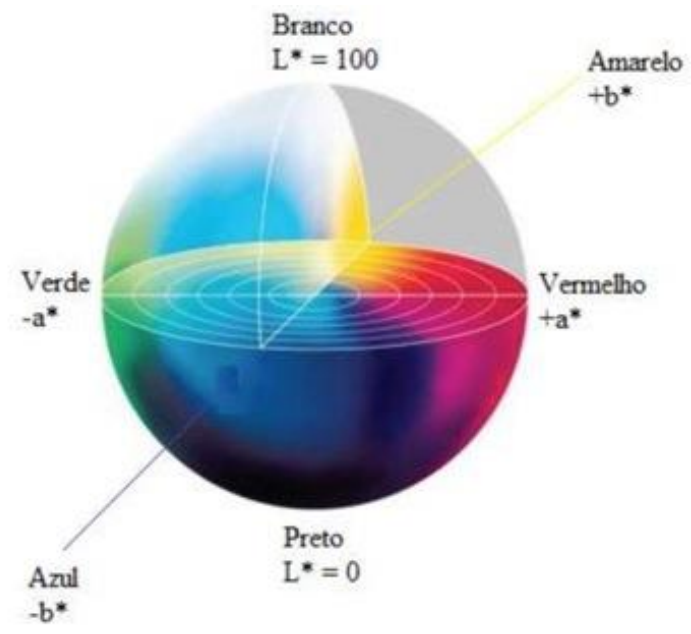

Fonte: adaptada de CIE (2018).

\section{Figura 5 - Identificação dos lenhos no corpo de prova}

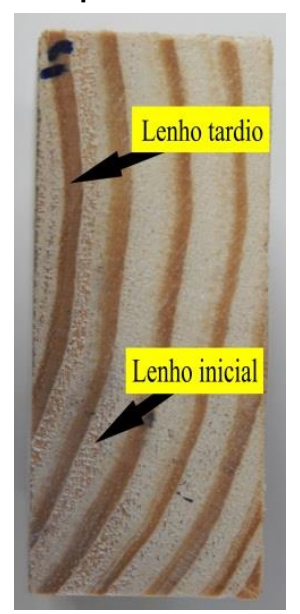

O modelo da Equação 3 foi idealizado por considerar os fatores individuais, bem como os efeitos de iteração entre duas variáveis.

Com relação às premissas da ANOVA, a normalidade dos resíduos foi avaliada com o auxílio do teste de normalidade de Anderson-Darling (ao nível de 5\% de significância); a homogeneidade das variâncias foi avaliada pelo gráfico de resíduos versus valores ajustados; e a independência foi investigada pelo gráfico de resíduos versus ordem de observação, sendo a qualidade dos ajustes medida pelo coeficiente de determinação $\left(\mathrm{R}^{2}\right)$.

Pela ANOVA do modelo de regressão é possível identificar os termos considerados significativos, assim como a ordem de significância deles. A significância dos termos do modelo foi avaliada pelo gráfico de Pareto.

\section{Resultados e discussão}

Na Figura 6 são ilustrados os valores médios, os intervalos de confiança da média (ao nível de 5\% de significância) e os coeficientes de variação $(\mathrm{CV})$ dos parâmetros colorimétricos $\left(\mathrm{L}^{*}, \mathrm{a}^{*}, \mathrm{~b}^{*}\right)$ e do número de lenhos (Nle) respectivamente. 
Figura 6 - Resultados dos parâmetros colorimétricos $L^{*}(a), a^{*}(b), b^{*}(c)$ e Nle (d)

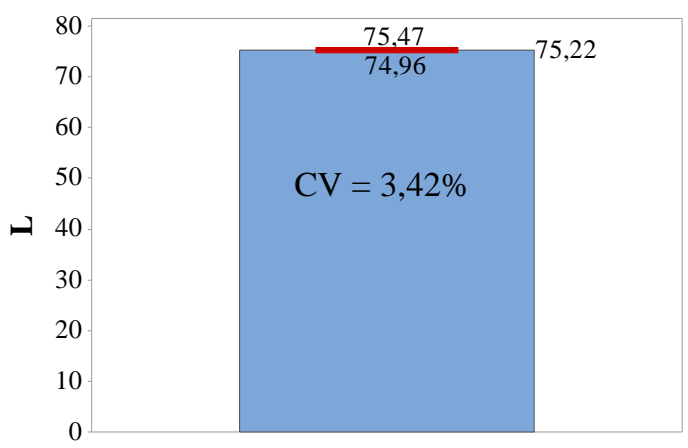

(a)

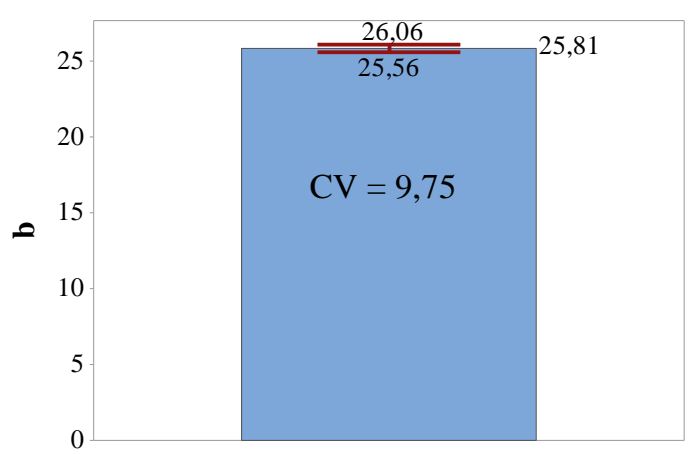

(c)

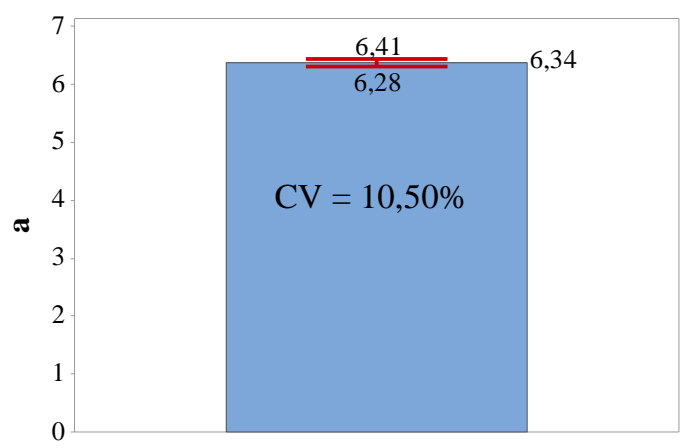

(b)

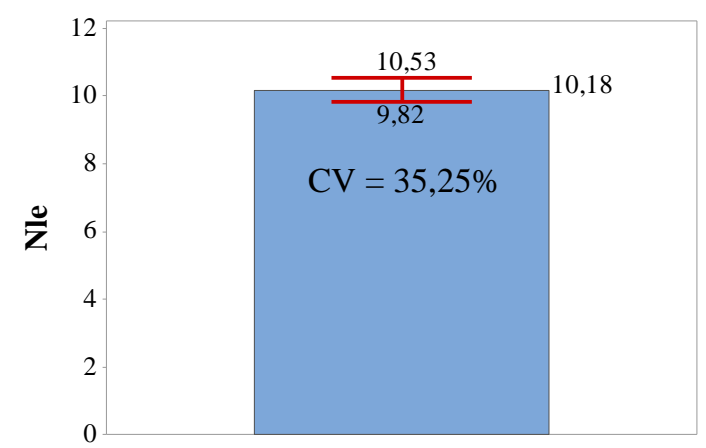

(d)

O valor médio determinado para o parâmetro $L^{*}$ neste estudo foi igual a 75,22, próximo ao determinado por outros autores (CONTE et al. 2014; PINCELLI; MOURA; BRITO, 2012; POUBEL et al. 2015) para a madeira de Pinus. De acordo com a classificação proposta por Camargos e Gonçalez (2001), o Pinus é uma madeira de coloração branco-amarelada e possui $\mathrm{L}^{*}$ superior a 54 . A variabilidade anatômica da madeira pode implicar valores diferentes para luminosidade, já que esse fator também pode influenciar a cor da madeira.

O valor médio para o parâmetro a* foi de 6,34, próximo ao determinado por Pincelli, Moura e Brito (2012) e ligeiramente superior ao determinado pelos demais autores que estudaram a madeira de Pinus (AMORIM; GONÇALEZ; CAMARGO, 2013; CONTE et al., 2014). Segundo Gierlinger et al. (2003), a coordenada cromática $\mathrm{a}^{*}$ é influenciada diretamente pelo teor de extrativos presentes na madeira.

Para o parâmetro $b^{*}$ o valor médio encontrado foi igual a 25,81, mesmo valor encontrado por Pincelli, Moura e Brito (2012) para a madeira de Pinus caribaea. Esse valor também está próximo ao determinado em outros estudos (CONTE et al., 2014; POUBELL et al., 2015). A pigmentação amarela é a principal responsável pela formação de cor na espécie de Pinus e está relacionada com a fotoquímica dos principais componentes da madeira, como a lignina e os extrativos (CONTE et al., 2014; PINCELLI; MOURA; BRITO, 2012).

A variabilidade dos anéis de crescimento é causada por diversos fatores, sendo o clima um dos que mais influencia, uma vez que os fatores climáticos contribuem para o maior ou menor crescimento das camadas de lenho inicial e tardio. Os anéis de crescimento, por sua vez, influenciam as diferentes características físicas e mecânicas da madeira.

Na Figura 7 são ilustrados o valor médio, o intervalo de confiança da média (ao nível de 5\% de significância) e o coeficiente de variação $(\mathrm{CV})$ do módulo de elasticidade $\left(\mathrm{E}_{\mathrm{c} 0}\right)$.

$\mathrm{O}$ valor médio para o $\mathrm{E}_{\mathrm{c} 0}$ da madeira de Pinus sp. neste estudo foi igual a $13.476 \mathrm{MPa}$ (com teor de umidade da ordem de 12\%), resultado próximo ao apresentado pela norma brasileira NBR $7190(\mathrm{ABNT}, 1997)\left(\mathrm{E}_{\mathrm{c} 0}=\right.$ $13.304,0 \mathrm{MPa})$ e por Trianoski et al. (2014) $\left(\mathrm{E}_{\mathrm{c} 0}=12.432,0 \mathrm{MPa}\right)$, ambos para a madeira de Pinus taeda $\mathrm{L}$. 
Figura 7 - Resultados do módulo de elasticidade

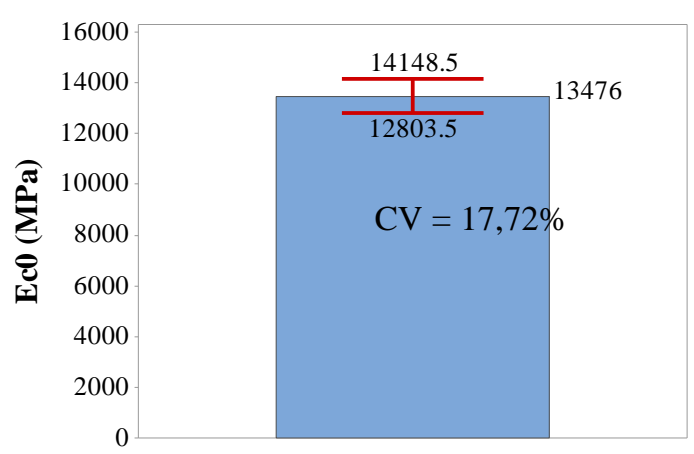

O modelo obtido via análise de regressão para a estimativa do módulo de elasticidade na compressão paralela às fibras das madeiras de Pinus sp. em função dos parâmetros colorimétricos e também do número de lenhos é apresentado na Equação 4, e na Figura 8 são apresentados os resultados das medidas de precisão da análise de regressão.

$\mathrm{E}_{\mathrm{c} 0}(\mathrm{MPa})=-258294+3608 \cdot \mathrm{L}-26449 \cdot \mathrm{a}+15431 \cdot \mathrm{b}+2441 \cdot \mathrm{Nle}+327 \cdot \mathrm{L} \cdot \mathrm{a}-205 \cdot \mathrm{L} \cdot \mathrm{b}-12.4 \cdot \mathrm{L} \cdot \mathrm{Nle}+33 \cdot \mathrm{a} \cdot \mathrm{b}+$ $86 \cdot \mathrm{a} \cdot \mathrm{Nle}-51.5 \cdot \mathrm{b} \cdot \mathrm{Nle}\left[\mathrm{R}^{2}=43,61 \%\right]$

Da Figura 8, a normalidade (P-valor $\geq 0,05$ - teste de Anderson-Darling), a homogeneidade (agrupamento dos valores ajustados em torno de 0) e a independência (inexistência de padrão na dispersão dos resíduos) dos resíduos foram atendidas, validando o modelo da ANOVA.

Da Equação 4, o valor do $\mathrm{R}^{2}$ obtido foi de apenas 43,61\%, entretanto, com toda a variabilidade intrínseca da madeira, constatou-se a significância do modelo com base nos resultados da ANOVA, o que possibilitou identificar a influência dos fatores e das interações, permitindo maior compreensão da relação entre as variáveis confrontadas.

Rall (2006) utilizou análise de regressão linear múltipla entre o módulo de elasticidade e o número de anéis por polegada e a proporção de lenho tardio para a madeira de Pinus taeda L., encontrando R ${ }^{2}$ igual a 41,5\%, bem próximo ao determinado neste estudo.

Do gráfico de Pareto (Figura 8d), apenas o fator Nle (número de lenhos) afetou de forma significativa os valores do módulo de elasticidade na compressão paralela (Equação 4), indicando pequena influência dos parâmetros colorimétricos, fato esse constatado na pesquisa de Amorim, Gonçalez e Camargo (2013), também com madeira de Pinus sp., pela fraca correlação entre as propriedades.

Ainda da Equação 4, aumentos no Nle implicam aumentos significativos nos valores do módulo de elasticidade na compressão paralela das madeiras de Pinus sp. Diversos autores relataram que o módulo de elasticidade é afetado pela presença de madeira juvenil, ou seja, madeira com menor quantidade de lenho tardio. Larson et al. (2001) afirmam que o módulo de elasticidade é correlacionado fortemente com a densidade, sendo influenciado pela quantidade de madeira juvenil. Ballarin e Palma (2003) encontraram diferenças de 54\% nos valores do módulo de elasticidade na madeira adulta em relação à juvenil da madeira de Pinus taeda. Barrios, Trincado e Watt (2017) encontraram coeficiente de correlação (r) de 52\% entre o módulo de elasticidade e a proporção de lenho tardio para madeira adulta de Pinus radiata D. Don.

Excluindo-se os termos não significativos no modelo da Equação 4, assim como expressa a Equação 5, o valor do coeficiente de determinação do modelo completo sofreu redução de apenas $3,11 \%$, o que reforça a pequena significância dos demais termos.

$\mathrm{E}_{\mathrm{c} 0}(\mathrm{MPa})=6339+72,2 \cdot \mathrm{Nle}\left[\mathrm{R}^{2}=40,50 \%\right]$

Eq. 5

Na Figura 9 são ilustrados o valor médio, o intervalo de confiança da média (ao nível de 5\% de significância) e o coeficiente de variação $(\mathrm{CV})$ da resistência à compressão paralela às fibras. 
Figura 8 - Resultados das medidas de precisão da análise de regressão para o módulo de elasticidade

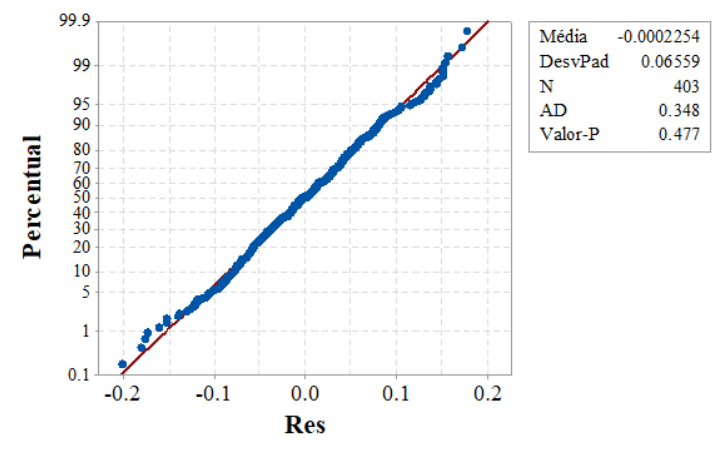

(a) Normalidade

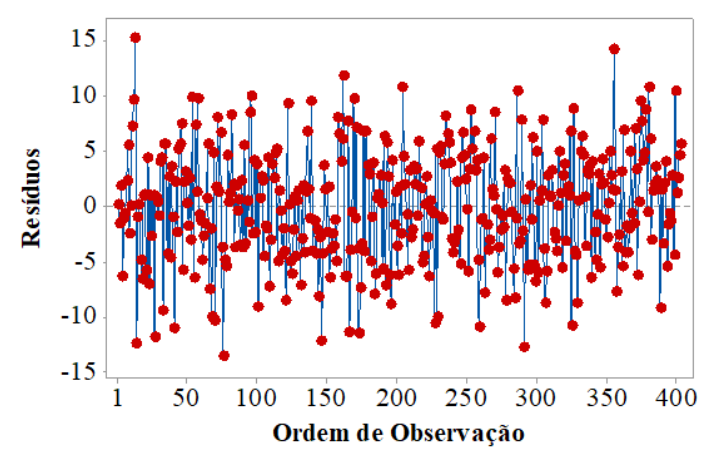

(c) Independência dos resíduos

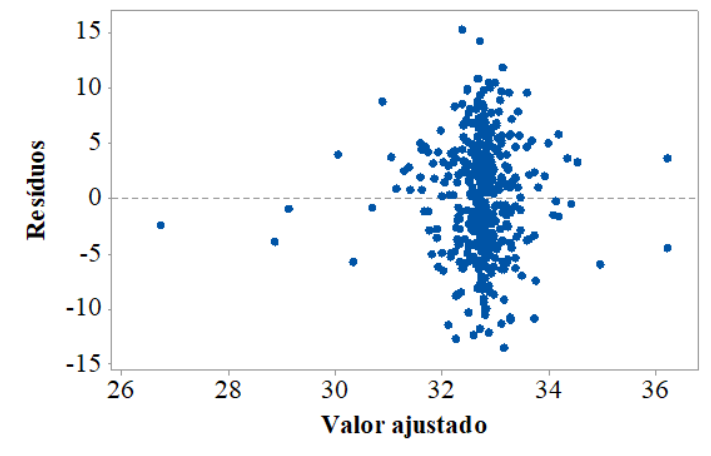

(b) Homogeneidade das variâncias

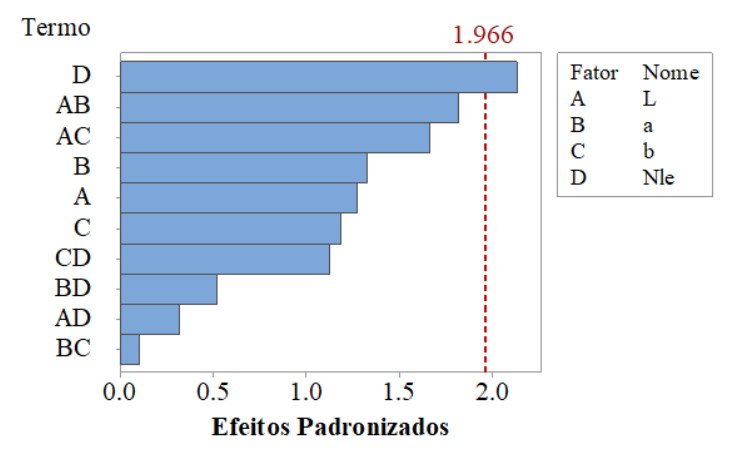

(d) Gráfico de Pareto

Figura 9 - Resultados da resistência na compressão paralela

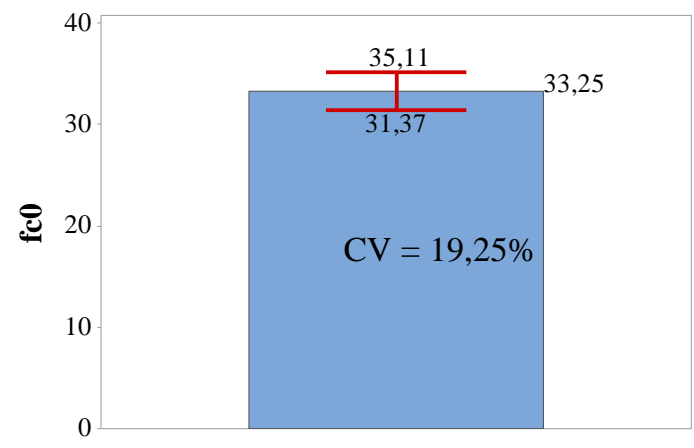

O CV obtido dos valores da resistência à compressão paralela às fibras foi de 19,25\%, próximo ao adotado pela norma brasileira NBR 7190 (ABNT, 1997) para a compressão paralela às fibras $(18 \%)$. O valor médio para a $\mathrm{f}_{\mathrm{c} 0}$ da madeira de Pinus sp. neste estudo foi igual a 33,25 $\mathrm{MPa}$ (com teor de umidade da ordem de $12 \%$ ), próximo ao apresentado pela norma brasileira para as espécies de Pinus caribea $\left(\mathrm{f}_{\mathrm{c} 0}=35,4 \mathrm{MPa}\right)$ e Pinus bahamensis $\left(\mathrm{f}_{\mathrm{c} 0}=32,6 \mathrm{MPa}\right)$. Segundo o IPT, a madeira de Pinus elliottii apresenta resistência à compressão paralela às fibras de $31,5 \mathrm{MPa}$ para umidade de $15 \%$.

A Equação 6 expressa a estimativa da resistência na compressão paralela às fibras das madeiras de Pinus sp. em função dos parâmetros colorimétricos e do número de lenhos, e na Figura 10 são apresentados os resultados das medidas de precisão da análise de regressão para a $\mathrm{f}_{\mathrm{c} 0}$.

$\mathrm{f}_{\mathrm{c} 0}(\mathrm{MPa})=352-2.53 \cdot \mathrm{L}-57.0 \cdot \mathrm{a}-2.8 \cdot \mathrm{b}+7.93 \cdot \mathrm{Nle}+0.380 \cdot \mathrm{L} \cdot \mathrm{a}-0.018 \cdot \mathrm{L} \cdot \mathrm{b}-0.041 \cdot \mathrm{L} \cdot \mathrm{Nle}+0.904 \cdot \mathrm{a} \cdot \mathrm{b}$

$+0.200 \cdot a \cdot$ Nle $-0.193 \cdot b \cdot$ Nle $\left[\mathrm{R}^{2}=48,53 \%\right]$ 
Figura 10 - Resultados das medidas de precisão da análise de regressão para a resistência na compressão

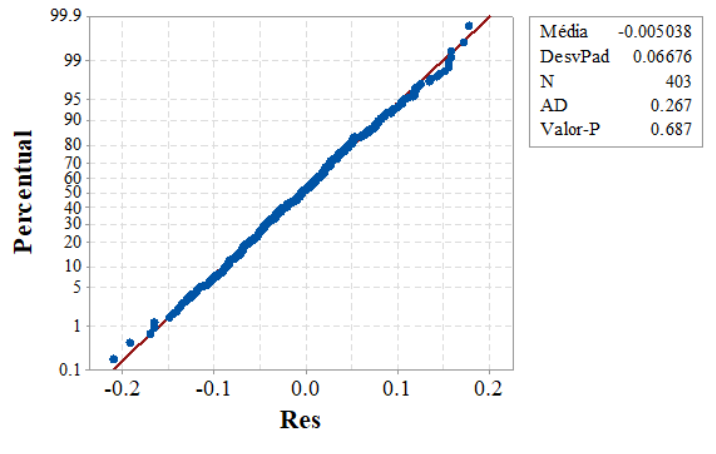

(a) Normalidade

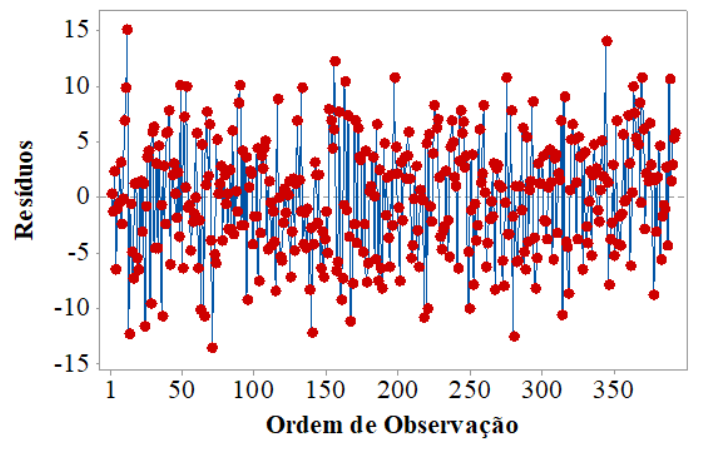

(c) Independência dos resíduos

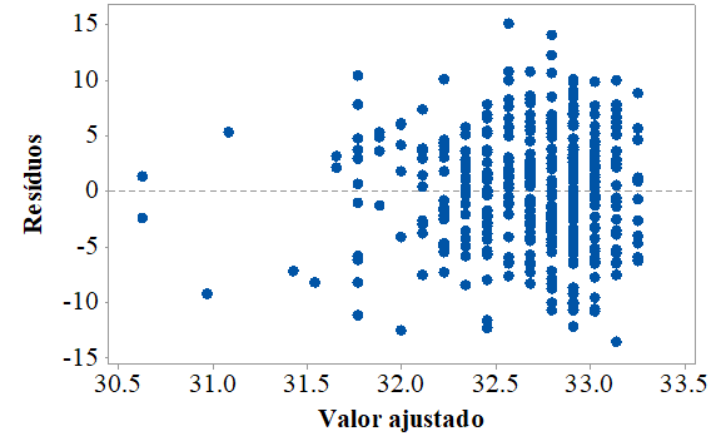

(b) Homogeneidade das variâncias

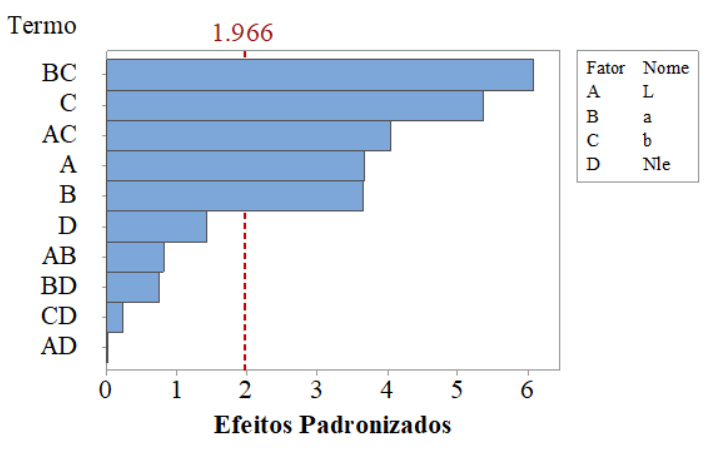

(d) Gráfico de Pareto

Da Figura 10, a normalidade (P-valor > 0,05 - teste de Anderson-Darling), a homogeneidade (agrupamento dos valores ajustados em torno de 0) e a independência (inexistência de padrão na dispersão dos resíduos) dos resíduos foram atendidas, validando o modelo da ANOVA. Do gráfico de Pareto, os fatores L*, a*, b* e as interações $L^{*} \cdot b^{*}$ e $a^{*} \cdot b^{*}$ afetaram de forma significativa os valores da $f_{c 0}$ (Equação 6), o que evidencia a pouca significância dos demais fatores avaliados.

Da Equação 6, o valor do $\mathrm{R}^{2}$ obtido foi de 48,53\%, entretanto, com toda a variabilidade intrínseca da madeira, a ANOVA acusou a significância do modelo, o que possibilitou identificar a significância dos fatores e das interações, permitindo maior compreensão da relação entre as variáveis confrontadas. Aumentos nos três parâmetros colorimétricos implicam reduções nos valores da resistência à compressão. A interação entre os fatores $\mathrm{L}^{*}$ e $\mathrm{b}^{*}$ implicou a redução dos valores da resistência, sendo constatados aumentos nessa propriedade pela interação dos fatores $a^{*}$ e $b^{*}$ (Figura 11).

Como apresentado anteriormente, a pigmentação amarela $\left(b^{*}\right)$ é a principal responsável pela formação de cor para o Pinus sp. e está relacionada com a fotoquímica dos principais componentes da madeira, como a lignina. A lignina é responsável, em parte, pela resistência mecânica da madeira (DUARTE, 2017).

Almeida (2017), em seu modelo de regressão para estimar a resistência à compressão paralela às fibras da madeira de tatajuba, cujo valor do parâmetro b* é igual a 23,85 (próximo ao determinado para o Pinus neste trabalho), obteve coeficiente de determinação de $72,49 \%$.

É importante ressaltar a escassez de trabalhos na literatura consultada que correlacionam propriedades físicas e mecânicas de Pinus sp. com seus parâmetros colorimétricos. Como não foi encontrada nenhuma pesquisa que correlaciona as propriedades mecânicas na compressão paralela às fibras com os parâmetros colorimétricos, não foi possível realizar a comparação dos resultados obtidos neste trabalho.

Figura 11 - Interação entre fatores para a resistência na compressão paralela às fibras -a) e (b) 


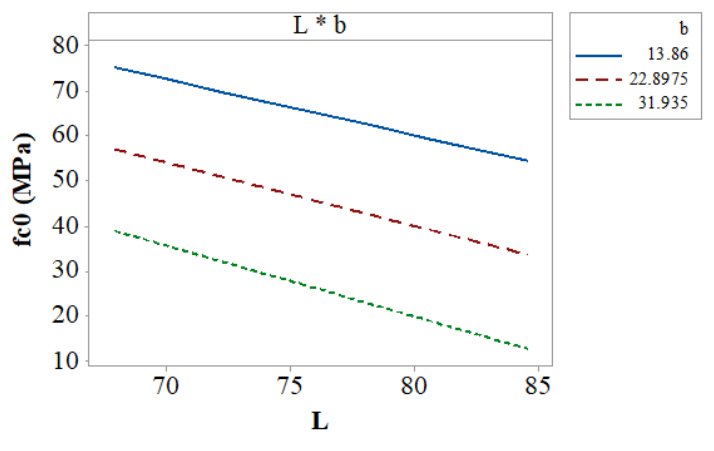

(a) $L^{*} \cdot b^{*}$

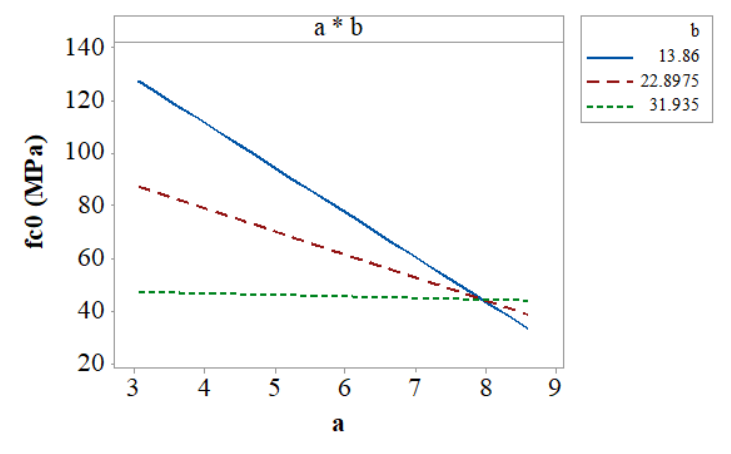

(b) $a^{*} \cdot b^{*}$

\section{Conclusões}

Os resultados da presente pesquisa permitem concluir que:

(a) a técnica de colorimetria aplicada à investigação de propriedades física e mecânica mostrou-se adequada, e os parâmetros colorimétricos $\left(\mathrm{L}^{*}, \mathrm{a}^{*} \mathrm{e} \mathrm{b}^{*}\right)$ utilizados nas definições do modelo são de fácil obtenção com o uso de um equipamento portátil (colorímetro);

(b) o Pinus sp. é uma madeira de coloração branco-amarelada, sendo a pigmentação amarela a principal responsável pela formação da cor neste gênero; o valor médio do parâmetro $L^{*}$ foi igual a 75,22; o valor médio determinado para o parâmetro a* foi igual a 6,34; e o parâmetro b* apresentou valor médio igual a 25,80 ;

(c) os valores dos $\mathrm{R}^{2}$ foram $40,50 \%$ para estimativa do módulo de elasticidade na compressão paralela às fibras e 48,53\% para estimativa da resistência à compressão paralela às fibras, o que restringe o uso das equações pelas pequenas precisões associadas, entretanto, com toda a variabilidade intrínseca da madeira, a ANOVA acusou a significância dos modelos, o que possibilitou identificar a influência dos fatores e das interações, permitindo maior compreensão da relação entre as variáveis confrontadas;

(d) o valor médio do $\mathrm{E}_{\mathrm{c} 0}$ foi igual a 13.681,87 MPa, a análise de regressão mostrou que apenas o Nle afetou significativamente os valores do $\mathrm{E}_{\mathrm{c} 0}$, e o aumento no número de lenhos gera aumento significativo nos valores do $\mathrm{E}_{\mathrm{c} 0}$; e

(e) o valor médio da resistência à compressão paralela às fibras foi igual a 33,25 MPa, adequado com os resultados da literatura, e a análise de correlação mostrou que apenas os fatores $\mathrm{L}^{*}, \mathrm{a}^{*}, \mathrm{~b}^{*}$ e as interações $L^{*} \cdot b^{*}, a^{*} \cdot b^{*}$ afetaram de forma significativa os valores da resistência na compressão paralela às fibras.

Novos modelos de regressão múltiplas devem ser propostos para estimativa de outras propriedades mecânicas da madeira de Pinus sp. em função dos parâmetros colorimétricos. Também deve ser avaliada a influência das características dos anéis de crescimento (número de anéis por polegadas, ângulo de inclinação e proporção de lenho tardio) nas propriedades físicas e mecânicas da madeira de Pinus sp. isentas de defeitos, o que possibilitará a geração de modelos com maior precisão.

\section{Referências}

ALMEIDA, D. H. Estimativa de propriedades de resistência e de rigidez de madeiras tropicais brasileiras pela técnica de colorimetria. São Carlos, 2017. 98 f. Tese (Doutorado em Estruturas e Construção Civil) - Universidade Federal de São Carlos, São Carlos, 2017.

ALMEIDA, T. H. et al. Density as estimator of dimensional stability quantities of Brazilian tropical woods. BioResources, v. 12, n. 3, p. 6579-6590, 2017.

AMORIM, P. G. R.; GONÇALEZ, J. C.; CAMARGOS, J. A. A. Propriedades da madeira de Pinus caribaea e Eucalyptus grandis estimadas por colorimetria. Cerne, v. 19, n. 3, p. 461-466, 2013.

ANDRADE JUNIOR, J. R. et al. Avaliação das estruturas de cobertura em madeira de um galpão de estoques de produtos químicos. Ambiente Construído, Porto Alegre, v. 14, n. 3, p. 75-85, jul./set. 2014. 
ASSOCIAÇÃO BRASILEIRA DE NORMAS TÉCNICAS. NBR 7190: projeto de estruturas de madeira. Rio de Janeiro, 1997.

BALLARIN, A. W.; PALMA, H. A. L. Propriedade de resistência e rigidez da madeira juvenile e adulta de Pinus taeda L. Revista Árvore, v. 27, n. 3, p. 371- 380, 2003.

BARCÍK, S.; GASPARÍK, M.; RAZUMOV, E. Y. Effect of thermal modification on the colour changes of oak wood. Wood Research, v. 60, n. 3, p. 385-396, 2015.

BARRIOS, A.; TRINCADO, G.; WATT, M. S. Wood properties of juvenile and mature wood of Pinus radiata D. Don trees growing on contrasting sites in Chile. Forest Science, v. 63, n. 2, p. 184-191, 2017.

BARROS, S. V. S.; MUNIZ, G. I. B.; MATOS, J. L. M. Caracterização colorimétrica das madeiras de três espécies florestais da Amazônia. Cerne, v. 20, n. 3, p. 337-342, 2014.

BERTOLINI, M. S. et al. Influência do comprimento de corpos de prova na obtenção do módulo de elasticidade $\mathrm{E}_{\mathrm{c} 0}$. Floresta e Ambiente, v. 19, n. 2, p. 179-183, 2012.

BURGER, M. B.; RITCHER, H. C. Anatomia da madeira. São Paulo: Nobel, 1991.

CADEMARTORI, P. H. G. et al. Colour responses of two fast-growing hardwoods to two-step steam-heat treatments. Materials Research, v. 17, n. 2, p. 487-493, 2014.

CAMARGOS, J. A. A.; GONÇALEZ, J. C. A colorimetria aplicada como instrumento na elaboração de uma tabela de cores de madeira. Brasil Florestal, v. 71, p. 30-41, 2001.

CONTE, B. et al. Propriedades físicas e colorimétricas da madeira termorretificada de Pinus elliotti var. elliotti. Scientia Forestalis, v. 42, n. 104, p. 545-553, 2014.

DUARTE, B. B. Influência de componentes químicos em propriedades mecânicas da madeira. São Carlos, 2017. 127 f. Tese (Doutorado em Ciência e Engenharia de Materiais) - Escola de Engenharia de São Carlos, Universidade de São Paulo, São Carlos, 2017.

EMPRESA BRASILEIRA DE PESQUISA AGROPECUÁRIA. [Home page]. 2018. Disponível em: https://www.spo.cnptia.embrapa.br. Acesso em: 7 jun. 2018.

FERREIRA, A. T. B. Caracterização da estrutura anatômica do lenho, dos anéis de crescimento e dos canais de resina de árvores de Pinus caribaea var. hondurensis Barr. Et Golf. Piracicaba, 2009. 83 f. Dissertação (Mestrado em Recursos Florestais) - Escola Superior de Agricultura Luiz de Queiroz, Universidade de São Paulo, Piracicaba, 2009.

FERRO, F. S. et al. Influência da posição dos instrumentos de medida na determinação do módulo de elasticidade da madeira na compressão paralela às fibras $\left(\mathrm{E}_{\mathrm{c} 0}\right)$. Revista Árvore, v. 39, n. 4, p. 743-749, 2015.

GARCIA, R. A. et al. Colorimetria de madeiras dos espécies Eucalyptus e Corymbia e sua correlação com a densidade. Cerne, v. 20, p. 509-517, 2014.

GARCIA, R. A.; MARINONIO, G. B. Variação da cor da madeira de teca em função da densidade e do teor de extrativos. Floresta e Ambiente, v. 23, n. 1, p. 124-134, 2016.

GIERLINGER, N. et al. Colour of larch heartwood and relationships to extractives and brown-rot decay resistance. Trees, Vancouver, v. 18, p. 101-108, 2003.

INSTITUTO DE PESQUISAS TECNOLÓGICAS. Informações sobre madeiras. 2017. Disponível em: http://www.ipt.br/consultas_online/informacoes_sobre_madeira. Acesso em: 7 jun. 2018.

INTERNATIONAL COMMISSION ON ILLUMINATION. COLORIMETRY — PART 4: CIE 1976 L*A*B* COLOUR SPACE. 2018 Disponível em: http://www.cie.co.at. Acesso em: 7 jun. 2018.

LARSON, P. R. et al. and properties of juvenile wood in southern pines: A synopsis. Madison: Forest Products Laboratory, 2001.

LAZAROTTO, M. et al. Resistência biológica e colorimetria da madeira termorretificada de duas espécies de Eucalipto. Revista Árvore, v. 40, n. 1, p. 135-145, 2016.

MEINTS, T. et al. Wood colour of central European wood species: CIELAB characterization and colour intensification. European Journal of Wood Products, v. 11, n. 2, p. 5155-5168, 2016. 
MONTES, C. S. et al. Genetic variation in wood color and its correlations with tree growth and density of Calycophyllum spruceanum at an early age in the Peruviam Amazon. New Forests, Dordrecht, v. 35, p. 5773, 2008.

PINCELLI, A. L. P. S. M; MOURA, L. F.; BRITO, J. O. Effect of thermal rectification on colors of Eucalyptus saligna and Pinus caribaea woods. Maderas, v. 14, n. 2, p. 239-248, 2012.

POUBEL, D. S.; GARCIA, R. A.; SANTOS, W. A.; LELIS, R. C. C.; VIEIRA, L. A. A. Análises físicas e colorimétricas da madeira de Pinus sp. modificada termicamente. Scientia Forestalis, v. 43, n. 107, p. 511521, 2015.

RALL, R. Influência das características dos anéis de crescimento na densidade, resistência e rigidez da madeira de Pinus taeda L. Botucatu, 2006. 73 f. Dissertação (Mestrado em Agronomia) - Faculdade de Ciências Agronômicas, Universidade Estadual Paulista “Júlio de Mesquita Filho”, Botucatu, 2006.

SANTINI, E. J.; HASELEIN, C. R.; GATTO, D. A. Comparative analysis of physical and mechanical properties old wood from three plantation softwoods. Ciência Florestal, v. 10, n. 1, p. 85-93, 2000.

TRIANOSKI, R. et al. Avaliação das propriedades mecânicas da madeira de espécies de Pinus tropicais. Scientia Forestalis, v. 42, n. 101, p. 21-28, 2014.

ZANUNCIO, A. J. V. et al. Química e colorimetria da madeira de Eucalyptus grandis W. Mill ex Maiden termorretificada. Revista Árvore, v. 38, n. 4, p. 765-770, 2014.

ZENID, G. J.; CECCANTINI, G. C. T. Identificação macroscópica de madeiras. São Paulo: IPT, 2007.

\author{
Lucas José Marini \\ Departamento de Engenharia Civil | Universidade Federal de São Carlos | Rod. Washington Luís (SP-310), Km 235, Jardim Guanabara | São \\ Carlos - SP - Brasil | CEP 13565-905 | Tel.: (19) 99179-9527 | E-mail: lucasjmarini@hotmail.com \\ Tiago Hendrigo Almeida \\ Instituto SENAI de Inovação Biomassa | Av. Angelina Tebet, Santa Luzia | Três Lagoas - MS - Brasil | CEP 79640-250 | Tel.: (67) 3919-2019 \\ | E-mail: tiago.henrique@gmail.com \\ Diego Henrique Almeida \\ Departamento de Engenharia Civil | Universidade Federal de Rondônia | BR 364, km 9,5 (sentido Acre) | Porto Velho - RO - Brasil | CEP \\ 76801-059 | Tel.: (69) 2182-2193 | E-mail: diegoestruturas@gmail.com \\ André Luis Christoforo \\ Departamento de Engenharia Civil | Universidade Federal de São Carlos | Tel.: (16) 3306-6828 | E-mail: christoforoal@yahoo.com.br \\ Francisco Antonio Rocco Lahr \\ Departamento de Engenharia de Estruturas | Universidade de São Paulo | Av. Trabalhador São Carlense, 400, Parque Arnold Schimidt | \\ Caixa Postal 359 | São Carlos - SP - Brasil | CEP 13566-590 | Tel.: (16) 3373-9483 | E-mail: frocco@sc.usp.br
}

\title{
Ambiente Construído
}

Revista da Associação Nacional de Tecnologia do Ambiente Construído Av. Osvaldo Aranha, $99-3^{\circ}$ andar, Centro

Porto Alegre - RS - Brasil CEP 90035-190

Telefone: +55 (51) 3308-4084

Fax: +55 (51) 3308-4054

www.seer.ufrgs.br/ambienteconstruido

E-mail: ambienteconstruido@ufrgs.br 